\title{
$\mathrm{SiO}_{2}$ and $\mathrm{ZnO}$ Dopants in 3D Printed TCP Scaffolds Enhances Osteogenesis and Angiogenesis in vivo
}

\author{
Gary Fielding and \\ W. M. Keck Biomedical Materials Research Laboratory School of Mechanical and Materials \\ Engineering Washington State University Pullman, WA 99164-2920, USA
}

\section{Susmita Bose}

W. M. Keck Biomedical Materials Research Laboratory School of Mechanical and Materials Engineering Washington State University Pullman, WA 99164-2920, USA Fax: (509) 335-4662 sbose@wsu.edu

\begin{abstract}
Calcium phosphate (CaP) scaffolds with three dimensionally (3D) interconnected pores play an important role in mechanical interlocking and biological fixation in bone implant applications. CaPs alone, however, are only osteoconductive (ability to guide bone growth). Much attention has been given to the incorporation of biologics and pharmacologics to add osteoinductive (ability to cause new bone growth) properties to $\mathrm{CaP}$ materials. Because biologics and pharmacologics are generally delicate compounds and also subject to increased regulatory scrutiny, there is a need to investigate alternative methods to introduce osteoinductivity to $\mathrm{CaP}$ materials. In this study silica $\left(\mathrm{SiO}_{2}\right)$ and zinc oxide $(\mathrm{ZnO})$ have been incorporated in to $3 \mathrm{D}$ printed $\beta$-tricalcium phosphate (TCP) scaffolds to investigate their potential to trigger osteoinduction in vivo. Silicon and zinc are trace elements that are common to bone and have also been shown to have many beneficial properties from increased bone regeneration to angiogenesis. Implants were placed in bicortical femur defects introduced to a murine model for up to 16 weeks. Addition of dopants into TCP increased the capacity for new early bone formation by modulating collagen I production and osteocalcin production. Neovascularization was found to be up to three times more than the pure TCP control group. The findings from this study indicate that the combination of $\mathrm{SiO}_{2}$ and $\mathrm{ZnO}$ dopants in TCP may be a viable alternative to introduce osteoinductive properties to CaPs.
\end{abstract}

\section{Keywords}

calcium phosphate; 3d printing; scaffold; silicon; zinc; in vivo; angiogenesis; osteogenesis

\section{Introduction}

Currently in the orthopedic and dental industry, calcium phosphate $(\mathrm{CaP})$ materials are amongst the most widely used and studied resorbable bone simulate due to their compositional similarity to natural bone and excellent biocompatibility. Presently, clinical utilization of $\mathrm{CaPs}$ can be found in a wide array of products including coatings on metallic implants, bone cements and as grafting materials [1]. $\beta$-tricalcium phosphate ( $\beta$-TCP) is a

(C) 2013 Acta Materialia Inc. Published by Elsevier Ltd. All rights reserved.

Publisher's Disclaimer: This is a PDF file of an unedited manuscript that has been accepted for publication. As a service to our customers we are providing this early version of the manuscript. The manuscript will undergo copyediting, typesetting, and review of the resulting proof before it is published in its final citable form. Please note that during the production process errors may be discovered which could affect the content, and all legal disclaimers that apply to the journal pertain. 
$\mathrm{CaP}$ material that has been of particular interest because its solubility product $\left(\mathrm{K}_{\mathrm{sp}}=1.25 \times\right.$ $10^{-29}$ ) allows the material to degrade over the course of months rather than several weeks such as a-TCP $\left(\mathrm{K}_{\mathrm{sp}}=3.16 \times 10^{-26}\right)$ or several years for hydroxyapatite $(\mathrm{HA})\left(\mathrm{K}_{\mathrm{sp}}=2.35 \times\right.$ $\left.10^{-59}\right)[2,3]$. This puts $\beta$-TCP in an ideal category for scaffolding material. New bone growth can occur faster than the dissolution of the scaffold, allowing the material to act as mechanical support for cellular migration, attachment and proliferation while the healing process takes place, but still achieving complete resorption relatively quickly so the healing process can complete.

Aside from biocompatibility, there are three major considerations that play a role in the success of a scaffold material for bone and dental implants: osteoconduction, osseointegration and osteoinduction. $\beta$-TCP is considered an osteoconductive material, or a material that permits bone growth onto its surface or throughout its pores. Osseointegration describes the ability to encourage mechanical interlocking between living tissue and the implanted device that can withstand functional loading [4]. This consideration is usually addressed by the incorporation of a complex porous structure within an osteoconductive implant. Many methods have been used to create scaffolding materials with controlled porosity including direct and indirect extrusion freeforming, selective laser sintering, stereolithography and ink-jet printing [5]. Direct ink jet printing has the benefits of very fine resolution, high degree of control over complex geometry and introduction of micro/macro porosity without the need for a sacrificial support structure [6-9]. Osteoinduction is generally described as the ability to stimulate primitive, undifferentiated and pluripotent cells to develop into the bone-forming cell lineage, or to put more simply, the ability to induce osteogenesis [10]. $\beta$-TCP, on its own, lacks osteoinductive capabilities and much time and effort has been spent improving this quality.

Many researchers have noted that physical material properties, such as the presence of microporosity, macroporosity and pore interconnectivity, may be enough to introduce osteoinductive capabilities [11-14], but most of the focus has been on incorporating pharmaceuticals and biologics to achieve this effect with varying degrees of success[2,1518]. While most studies have noted markedly positive effects with pharmaceutical incorporation, the compounds tend to be fragile, making commercial sterilization difficult, and usually only exhibit short term release profiles. It is also important to note extreme hesitation by the FDA to approve combination products containing biologics. An alternative of interest to this strategy is to incorporate small amounts of biologically relevant metal oxide dopants into TCP in place of the pharmaceuticals. Our research and others have noted that the use of dopants such as silicon, zinc, strontium and magnesium have the ability to not only tailor strength and strength degradation, but also enhance the biological response in vitro and in vivo[19-23]. Silicon has been noted to be an important trace element in osteogenesis, with research results indicating a strong stimulatory effect on cellular activities such as proliferation, differentiation, and mineralization of osteoblast cells as well as facilitating osteogenic differentiation of mesenchymal stem cells [24-26]. Other studies have observed that with supplemental silicon and monomethyl trisilanol in osteoporotic patients increased the femoral and lumbar spine bone mass density and was found to be more effective than a bisphosphonate and sodium fluoride treatment [27]. Botelho, et al. also noted that the presence of silicon in calcium phosphates increased alkaline phosphatase activity and total protein production of osteoblasts in vitro [28]. Zinc, also considered an essential trace element, is released during the skeletal breakdown process and has demonstrated the ability to inhibit osteoclastic bone resorption [29,30]. It also boosts osteogenic characteristics by inducing osteoblastogenesis as well as osteoblastic differentiation and mineralization $[31,32]$. Other studies have demonstrated that postmenopausal osteoporotic patients exhibit lower levels of skeletal zinc than control groups [33]. 
In this study we have combined the osteoconductive capabilities of $\beta$-TCP, added the capacity for osseointegration through complex geometry design and 3D printing and supplemented the material with metal oxide dopants silica $\left(\mathrm{SiO}_{2}\right)$ and zinc oxide $(\mathrm{ZnO})$ to promote osteoinduction. Scaffolds were implanted into a murine femoral defect model and analyzed over the course of 16 weeks for osteogenic properties.

\subsection{Materials and Methods}

\subsection{Fabrication of Porous Scaffolds}

High purity oxide based sintering additives, silicon dioxide ( $\mathrm{SiO} 2)(99 \%+$ purity) and zinc oxide ( $\mathrm{ZnO})(99.9 \%+$ purity) were purchased from Fisher Scientific (Fair Lawn, NJ). Synthetic $\beta$-tricalcium phosphate powder was obtained from Berkley Advanced Biomaterials Inc. (Berkeley, CA) with an average particle size of $550 \mathrm{~nm}$ and specific average surface area of $10-50 \mathrm{~m}^{2} \mathrm{~g}^{-1}$. Powder was made in batches of $100 \mathrm{~g}$ and doped with $0.25 \mathrm{wt} \% \mathrm{ZnO}$ and $0.5 \mathrm{wt} \% \mathrm{SiO}_{2}$ by adding dopant powder to $\beta$-TCP precursor powder. Dopant amounts were chosen based on previous research from our group that optimized concentration based on cell interaction as well as physicochemical properties $[9,20,21,34]$. Powder was added to and mixed in $500 \mathrm{~mL}$ polypropylene Nalgene bottles, with $300 \mathrm{~g}$ of 5 $\mathrm{mm}$ diameter zirconia milling media. $150 \mathrm{~mL}$ of ethanol was added and ball milling was carried out for $6 \mathrm{~h}$ at $70 \mathrm{rpm}$ to minimize the formation of agglomerates and increase the homogeneity of the powders. After milling, the Nalgene bottle was placed in an oven at $60^{\circ}$ $\mathrm{C}$ for $24 \mathrm{~h}$ for drying. Milling media were removed and the powder was further dried at $60^{\circ}$ $\mathrm{C}$ for another $24 \mathrm{~h}$. Finally, agglomerates were removed using a mortar and pestle.

Cylindrical scaffold CAD files (diameter $3.3 \mathrm{~mm}$ and height $5 \mathrm{~mm}$ ) were created with interconnected square channels of $423 \mu \mathrm{m}$, with the expectation of roughly $30 \%$ shrinkage after sintering for a final pore size of $300 \mu \mathrm{m}$ (Figure 1). Scaffolds were fabricated using a 3D printer (R-1 R\&D printer by ProMetal) by a process described previously [9]. Briefly, the printer head sprays binder onto loose powder in the deposition bed according to a CAD pattern, the deposition bed is lowered by $20 \mu \mathrm{m}$ and the feeder bed is raised by $60 \mu \mathrm{m}$. The spreader will then push the excess powder from the feeder bed and evenly spread it onto the lowered deposition bed. The process is repeated, layer by layer, until the CAD image is completely printed. After cleaning, green scaffolds were sintered in a muffle furnace at $1250^{\circ} \mathrm{C}$ for $2 \mathrm{~h}$. Sintered samples generally had a shrinkage rate of around $25 \%$, giving final dimensions of $2.5 \mathrm{~mm}$ diameter and $3.75 \mathrm{~mm}$ height with a final pore size of $317 \mu \mathrm{m}$. A previous study demonstrated that the addition of $\mathrm{SiO}_{2}$ and $\mathrm{ZnO}$ decreased a-phase formation in the sintered scaffolds and had as much as a 2.5 times increase in compressive strength when compared to the pure samples [9]. Samples were sterilized by autoclaving at $121{ }^{\circ} \mathrm{C}$ for 20 minutes.

\subsection{Implantation Procedure}

Sprague-Dawley rats (280-300 g, Charles Rivers Laboratories International, Inc., Wilmington, MA, USA) were used as an animal model for this study. Prior to surgery, the rats were housed in individual cages with alternating $12 \mathrm{~h}$ cycles of light and dark in temperature- and humidity-controlled rooms. Ethics approval for animal experimentation was obtained from Washington State University. Following acclimatization, all animals underwent bilateral surgery to create a bicortical defect in the distal femur on the popliteal plane ( $2.5 \mathrm{~mm}$ diameter). This defect model was designed by trained veterinary surgeons and found previously to be critical in size [23]. Rats were anesthetized using IsoFlo (isoflurane, USP, Abbott Laboratories, North Chicago, IL, USA) coupled with an oxygen (Oxygen USP, A-L Compressed Gases Inc., Spokane, WA, USA) regulator, and monitored by pedal reflex and respiration rate to maintain proper surgical anesthesia. The defect was 
created in either femur, proximal to the lateral condyle, by means of 1-3 mm drill bits. The cavity was rinsed with physiological saline to wash away remaining bone fragments. Each animal received a control implant (pure TCP) and in addition, a doped implant in the contralateral leg. Following implantation, undyed braided-coated polyglycolic acid synthetic absorbable surgical suture (Surgical Specialties Corporation, Reading, PA, USA) was used for stitching. Disinfectant was applied to the wound site to prevent infection. At 4,6, 8, 12 and 16 weeks post-surgery, rats with implants were euthanized by overdosing with halothane in a bell jar, followed by administration of a lethal injection of potassium chloride (70\%) into the heart. For a control group, similar surgical procedures were undertaken but only one implant was placed (either pure or doped). Controls were used for urine analysis throughout the 16 week study. Overnight urine samples were collected in metabolic cages at: $0,2,8,14,21,42,63,84$ and 112 days after surgery. A total of 24 rats were used for the experimental group ( 4 for each time point for $4,6,8$ and 12 weeks) and 8 rats for the control group (4 of each composition at week 16).

\subsection{Mechanical Pushout Analysis}

Mechanical testing was performed at 4 weeks after implantation. At test jig was manufactured to press the samples out of the bone using a screw driven universal testing machine (AG-IS, Shimadzu, Japan) at a constant crosshead speed of $0.33 \mathrm{~mm} / \mathrm{min}$.

\subsection{Histomorphology}

The bone-implant specimens were fixed in $10 \%$ neutral buffered formalin solution and dehydrated in graduated ethanol (70\%, 95\% and 100\%) series. After embedding samples in Spurr's resin, each undecalcified implant block was sectioned perpendicular to the implant surface using a low-speed diamond blade. After polishing, the sections were stained by Goldner- Masson trichrome stain and observed by light microscopy (Olympus BH-2, Olympus America Inc., USA).

\subsection{Immunohistochemistry}

For the immunohistochemical work scaffolds, along with surrounding tissue, were excised and fixed in $4 \%$ paraformaldehyde followed by decalcification in 10\% EDTA. Samples were then dehydraded in a graded ethanol series and embedded in paraffin and sectioned to $10 \mu \mathrm{m}$ thickness with a standard microtome and placed on glass slides. Afterwards, sections were deparaffinized with xylene and rehydrated to water in a graded ethanol series. Antigen retrieval was performed in a Tris-EDTA Buffer $(10 \mathrm{mM}$ Tris Base, $1 \mathrm{mM}$ EDTA Solution, $0.05 \%$ Tween $20, \mathrm{pH} 9.0$ ) by use of a vegetable steamer for 20 minutes followed by rinsing for 10 minutes in cold running tap water. Slides were then washed $2 \times 5$ minutes in TBS (Tris-buffered saline, $250 \mathrm{mM} \mathrm{NaCl}, \mathrm{pH} 8.3$ ) plus $0.025 \%$ Triton $\mathrm{X}-100$ and blocked in $10 \%$ normal serum with $1 \%$ BSA in TBS for 2 hours at room temperature. Slides were drained and primary antibody, either for collagen type 1 (COL1), osteocalcin (OCN) (Abcam, Cambridge, MA), or vonWillebrand Factor (vWF) (Millipore, Billerica, MA) diluted in TBS with $1 \%$ BSA (1:100 dilution) was added and set to incubate overnight at $4^{\circ} \mathrm{C}$. The samples were then rinsed $2 \times 5 \mathrm{~min}$ in TBS with $0.025 \%$ Triton. The secondary antibody, goat anti-rat Oregon green 488 (Molecular Probes, Eugene, OR), was diluted 1:100 in TBS and was used to incubate the samples with COL1 and OCN for $1 \mathrm{~h}$. After rinsing three times for 10 minutes each with TBS the samples were mounted with ProLong Gold Antifade Reagent with DAPI (Life Technologies, Grand Island, NY), coverslipped and set to cure. Afterwards, confocal micrographs were taken using a Zeiss 510 laser scanning microscope (LSM 510 META, Carl Zeiss MicroImaging, Inc., NY, USA) with excitation wavelength of $488 \mathrm{~nm}$ and emission wavelength of $540 \mathrm{~nm}$. The vWF samples were further processed with a blood vessel staining kit (Millipore, Billerica, MA) by incubating with premade secondary goat anti-rabbit antibody for $15 \mathrm{~m}$, rinsing with TBS rinse buffer and reacting with streptavidin- 
HRP for 15 minutes. Samples were rinsed again and incubated in pre-prepared chromogen reagent for 10 minutes. Slides were then dehydrated through an ethanol grade mounted in a xylene based mounting fluid and a coverslip was applied. Samples were observed by light microscopy (Olympus BH-2, Olympus America Inc., USA).

\subsection{FESEM}

After sample excision, specimens were fixed in $10 \%$ neutral buffered formalin solution. Post-fixation was performed with $2 \%$ osmium tetroxide $\left(\mathrm{OsO}_{4}\right)$ for $2 \mathrm{~h}$ at room temperature. The fixed samples were then dehydrated in an ethanol series (30\%, 50\%, 70\%, 95\% and $100 \%$ three times), followed by a hexamethyldisilane (HMDS) drying procedure. After drying, the samples were immersed in liquid nitrogen and the implant was freeze fractured. Samples were then gold sputter coated and observed under field emission scanning electron microscope (FESEM) (FEI 200F, FEI Inc., OR, USA). Additionally, another set of samples was extracted and fixed in $10 \%$ neutral buffered formalin solution for 24 hours. After fixation, samples were cut longitudinally in half using a low speed diamond saw at 600 RPM. Halves were placed in glass vials with $1 \mathrm{mg} / \mathrm{mL}$ Proteinase $\mathrm{K}$ in a $10 \mathrm{mg} / \mathrm{mL}$ sodium dodecyl sulfate (SDS) in distilled water solution to digest any organic material. Vials were placed in an incubator at $37^{\circ} \mathrm{C}$ with gentle agitation for 1 week. The Proteinase K solution was changed every 24 hours. After one week, samples were rinsed with distilled water 3 times to remove any remaining debris. Acetone was then added to the samples and let sit for 3 hours. After 3 hours, acetone was replaced and let sit overnight. Acetone was removed and samples gently shaken to remove excess solution. Diethyl ether was then added to the sample and let sit for 8 hours, then subsequently changed with fresh ether and let sit overnight. The ether solution was then discarded and samples shaken to remove any residue, then sample were placed in oven at $60^{\circ} \mathrm{C}$ for 8 hours on a glass dish to evaporate any remaining residue. Finally, samples were gold sputtered and imaged using back scattered electrons (BSE) on the FESEM.

\subsection{TRAP Staining}

Samples were evaluated for tartrate resistant acid phosphatase (TRAP) activity. Paraffin embedded sections were deparaffinized and rehydrated as described previously and slides were placed in $1 \%$ Naphthol AS-BI Phosphate (0.05M Napthol AS-BI phosphate in 2ethoxyethanol) stock incubation medium (3\% glacial acetic acid, $0.1 \mathrm{M}$ sodium acetate, $0.05 \mathrm{M}$ sodium tartrate) for 1 hour at $37^{\circ} \mathrm{C}$. Next slides were transferred to the developing solution ( $2 \% 0.5 \mathrm{M}$ sodium nitrate solution, $2 \%$ basic fuchsin solution $(0.15 \mathrm{M}$ basic fuchsin in $2 \mathrm{~N} \mathrm{HCL}$ ) in stock incubation medium) and kept at $37^{\circ} \mathrm{C}$ for $5-12 \mathrm{~m}$. Slides were then rinsed with water 3 times and counterstained with $0.02 \%$ Fast Green for 30 s. Finally, slides were dehydrated through an ethanol grade and cleared in xylene and observed by light microscopy (Olympus BH-2, Olympus America Inc., USA).

\section{$2.8 \mathrm{Ca}^{2+}, \mathrm{Zn}^{2+}$, and $\mathrm{Si}^{4+}$ ion excretion}

$\mathrm{Ca}^{2+}, \mathrm{Zn}^{2+}$ and $\mathrm{Si}^{4+}$ content in urine samples was measured using a Shimadzu AA-6800 atomic absorption spectrophotometer (Shimadzu, Kyoto, Japan). Standard solutions were freshly prepared in ionization buffer to obtain a final concentration of $1-20 \mu \mathrm{g} \mathrm{ml}^{-1}$. Calcium, zinc, silicon and ionization buffer standards were purchased from High-Purity Standards (Charleston, SC, USA).

\subsection{Histomorphometry}

Histomorphometry for samples stained with Goldner's Trichrome was performed according to a protocol developed by Egan, et al. [35] with 3 random areas of interest in 3 different samples. For samples that were examined using fluorescent microscopy, images were first 
converted to grayscale. Image $\mathbf{J}$ (http://imagej.nih.gov/ij/) was then used to measure the area, integrated density and mean gray value after calibrating for microscope used to take the pictures. The corrected total fluorescence $(\mathrm{CTCF})$ was taken as: $\mathrm{CTCT}=$ Integrated Density - (Area of micrograph $\times$ mean fluorescence of background readings). At least 3 random areas of interest in 3 different samples were used for each group.

\subsection{Statistical Analysis}

A one sided paired t-test was used to evaluate statistical differences between samples from the control group and doped group. Due to the limited availability of samples that is associated with an animal model study, results were found to be significant at $\mathrm{p}<0.1$, with at least $\mathrm{n}=3$ samples.

\subsection{Results}

\subsection{Mechanical Pushout Analysis}

Mechanical pushout testing was performed at 4 weeks. Full interlocking was achieved at this time and the bone was broke in each case before the implant loosened from the defect site for both the pure and doped samples. Due to this result, further time points were not considered for testing.

\subsection{New Bone Growth}

At 6 and 8 weeks, Goldner's trichrome staining and subsequent histomorphological measurements reveal enhanced bone growth in samples doped with $\mathrm{SiO}_{2} / \mathrm{ZnO}$ (Figure 2). By 12 weeks, samples had nearly complete infiltration of new bone and a distinction between the samples was difficult to discern. Neither sample indicated a difference in degradation kinetics of the calcium phosphate structure over the course of 12 weeks.

\subsection{Collagen I Formation}

Samples doped with $\mathrm{SiO}_{2} / \mathrm{ZnO}$ developed significantly increased collagen I formation at week 6 (Figure 3). The collagen levels measured in doped samples remained relatively stable until week 16, where a noticeable increase was observed. The pure samples also had a relatively stable amount of collagen formation over the first 12 weeks with an increase at week 16. Although not statistically significant, the average collagen I formation in doped samples was greater than that of the pure samples at weeks 12 and 16 .

\subsection{Osteocalcin Expression}

The expression of osteocalcin was measured at weeks 6, 8, 12 and 16 (Figure 4). The doped samples, on average, had increased amounts of osteocalcin protein present in samples at weeks 6,8 and 12 with statistical significance for weeks 8 and 12 when compared to pure TCP scaffolds. For both sets of samples, levels remained relatively high and constant over the first 12 weeks and then there was a noticeable drop in activity at week 16.

\subsection{Scaffold Dissolution}

Figure 6 shows BSE imaging of longitudinal halves of implants after 6, 8 and 12 weeks. At the 6 week time point, both pure and doped samples retained most of their structural characteristics. By 8 weeks, however, the pure samples began to demonstrate increased degradation properties when compared to the doped samples, a trend that continued through the 12 week observation perior. At 12 weeks, pure samples exhibited significant degradation when compared to the $\mathrm{SiO}_{2} / \mathrm{ZnO}$ doped samples. 


\subsection{New Blood Vessel Formation}

Figure 7 shows results from vWF immunohistostaining. Samples containing $\mathrm{SiO}_{2} / \mathrm{ZnO}$ had remarkably higher number of new blood vessel formation than their pure counterparts at weeks 6,8 and 12 . By the $16^{\text {th }}$ week, however, samples revealed nearly identical total blood vessel formation. Staining also suggest larger vessel and more complex vessel formation in the doped samples. FESEM results (Figure 8) confirm what was observed with the vWF staining. Blood vessel formation in the doped samples was more prevalent than in the pure samples and vascular branching morphogenesis was more notable.

\subsection{Osteoclast Activity}

TRAP staining (Figure 9) was performed to discern differences in osteoclastic activity between the pure and doped samples. Both pure and doped samples demonstrated similar TRAP activity through the 16 week study.

\subsection{Ionic Excretion}

AAS results (Figure 10) indicate no perceivable difference in scaffold degradation throughout the 16 week study. Urine $\mathrm{Ca}^{2+}$ levels measured indicate no trend over the course of the study, but animals containing pure or doped samples essentially had equal urine concentrations of $\mathrm{Ca}^{2+}$ throughout. Results from $\mathrm{Si}^{4+}$ analysis also indicate no difference between the control and doped implant, but demonstrate a slight increase in urine $\mathrm{Si}^{4+}$ levels immediately after surgery. $\mathrm{Zn}^{2+}$ concentration in urine again did not have any differences between the control and doped group, but showed a slight decrease in concentration immediately following implantation.

\subsection{Discussion}

The key reasons for introducing porosity into calcium phosphate bone void fillers are for enhanced nutrient delivery and mechanical interlocking at the bone-implant interface as well as to facilitate bone ingrowth. Results from the pushout tests at 4 weeks indicate a very rapid interlocking effect between the implant and the bone. While it was expected that, at 4 weeks, there would have been some resistance to push the implant free, it was unanticipated that complete fusion would have already taken place. Microporosity, macroporosity, pore size and pore shape all play important roles in the joining of the bone-implant boundary $[13,36,37]$. Studies have demonstrated that anywhere between $100 \mu \mathrm{m}$ and $1000 \mu \mathrm{m}$ is sufficient for bone ingrowth [38]. Previous studies by our group show that the 3D printing method used incorporates microporosity as well as the designed macroporosity into the samples $[9,39]$. These previous results indicated that the pure samples had an average of $15.64 \%$ microporosity (open pores only) and the $\mathrm{Si} / \mathrm{Zn}$ doped samples $28.25 \%$ microporosity (open pores only) along with the $35.5 \%$ designed macroporosity. The availability of micropores $(<50 \mu \mathrm{m})$ in a macroporous scaffold has been shown to drastically increase bone infiltration [40]. The micropores on the surface of the implant are faster for bone to fill (less void area), while the macropores take much longer (much larger void area). Essentially, the micropores allow for rapid mechanical interlocking, while interconnected macropores allow for nutrient delivery and cell migration and proliferation to the center of the scaffold. In this case, the micropores allowed this effect, acting as adhesion points at the bone-implant boundary causing the rapid interlocking effect between the host tissue and $\mathrm{CaP}$ implant. Trichrome staining (Figure 2) results at 6 weeks confirm that both pure and doped samples had complete infiltration of mineralized bone into the micropores at the boneimplant interface. The doped samples at week 6 , however, also showed new bone tissue growth into some of the macropores as well and histomorphometry showed a significant increase in new bone formation when compared to the pure samples. At week 12, both samples demonstrated the progression of tissue integration throughout the scaffold, filling 
both the micropores as well as the macropores, with doped samples having significantly higher bone formation. By 12 weeks, both samples showed nearly complete infiltration of mineralized bone tissue and differences were difficult to detect. Because micropore and macropore characteristics between the doped and pure samples were essentially the same, the increased rate of bone regeneration is likely to be linked to the incorporation of the $\mathrm{SiO}_{2} /$ $\mathrm{ZnO}$ dopant combination.

In a study performed by Hadley et al. [41], dietary zinc was linked to inhibition of osteoclastic resorption and increased markers for osteoblast differentiation, matrix maturation and mineralization in long bones of growing rats. Zinc deficiency, in other studies, has been linked to the suppression of matrix mineralization and osteoblastic differentiation [31,42]. Silicon has been linked to increased bone mineralization [43], increased bone growth $[19,44]$ as well as increased osteoblastic cell differentiation [24,45,46]. By incorporating $\mathrm{ZnO}$ and $\mathrm{SiO}_{2}$ into the $\mathrm{CaP}$ scaffolds, an effective delivery system of $\mathrm{Zn}^{2+}$ and $\mathrm{Si}^{4+}$ has been achieved as indicated by results from this study. Collagen type I is the dominant fibrous protein in hard tissues such as bone and dentin. Osteoblasts, during the proliferation and maturation phase of their lifecycle, will produce a collagen extracellular matrix. This matrix will then become mineralized at the behest of various signaling mechanisms, most notably bone sialoprotien, ostopontin and osteocalcin [47]. It has been reported that collagen I mRNA levels are not effected by dietary zinc administration $[41,48]$ in various animal models, but higher levels of collagen I production were seen in this study, significantly so at week 6 (Figure 3). It is possible that greater amount of collagen I was due to increased bone infiltration into the scaffolds, rather than increased production of collagen. However, if correlated with Trichrome data (Figure 2), the data does not seem to match this scenario well. Seaborn and Nielsen [49] demonstrated that $\mathrm{Si}^{4+}$ can play an important role at different stages of bone healing and, more specifically, silicon deprivation decreases the formation of collagen associated with wound healing. Results from this study indicate a high level of collagen I during the initial healing phase in samples containing $\mathrm{SiO}_{2} / \mathrm{ZnO}$ and a steady production up until the 12 week time point and then a large increase. The pure samples saw a steady increase in collagen I over the course of the study. Overall, then, the pure samples seem to have more collagen I formation as more new bone tissue was forming, while the doped samples exhibited behavior of a $\mathrm{Si}^{4+}$ modulated response.

Osteocalcin is considered to be the latest expression marker in mature osteoblasts and is thought to trigger the mineralization process [50]. In this study, both the pure and doped scaffolds exhibited similar trends in osteocalcin expression during the 16 week study (Figure 4). At 6 weeks, levels were high and remained so through 12 weeks. At 16 weeks there was a noticeable drop in osteocalcin presence. The doped samples maintained higher levels at 6, 8 and 12 weeks and identical levels when compared to pure samples at week 16. Although studies have revealed that both $\mathrm{Si}^{4+}$ and $\mathrm{Zn}^{2+}$ can effectively upregulate osteocalcin activity [46,51], the mechanism of action is poorly understood. Varanasi et al. [52] proposed that it may be a downstream effect of increased collagen I levels. Briefly, osteoblasts will bind to the mature collagen matrix, which is $\alpha_{2} \beta_{1}$ integrin mediated process. Integrin binding at the osteoblast cell membrane upregulates the expression of mitogen-activated protein kinases (MAPK). MAPK transduces signals to the cell nucleus and phosphorylate to activate alkaline phosphatase (ALP) and runt related transcription factor 2 (Runx2). ALP and Runx2 will then bind to the promoter region of genes such as osteocalcin. A simplified cartoon is presented in Figure 5. Previous studies have demonstrated that the addition of $\mathrm{SiO}_{2}$ and $\mathrm{ZnO}$ in these amounts actually increase densification by significantly decreasing the alpha phase formation at high sintering temperatures when compared to pure samples, leading to slower degradation kinetics $[9,21,22]$. Observation utilizing backscatter SEM, Figure 6. also noted significantly higher degradation in the pure samples over 12 weeks. As osteoblasts have 
demonstrated significant positive response to the dissolution products of $\mathrm{CaPs}\left(\mathrm{PO}_{4}^{3-}\right.$ and $\left.\mathrm{Ca}^{2+}\right)[46,53]$ and the pure samples are significantly more soluble due to alpha phase formation, the increased osteogenic effects observed in the doped samples, then, is very likely due to the presence of silicon and zinc.

Another important factor that affects the rate of new bone growth is angiogenesis. In order to meet the nutritional demands of new bone growth, a vascular network has to be present. In the present study, there is a stark difference in new blood vessel formation as observed by vWF staining (Figure 7) between the pure and doped scaffolds over the first 12 weeks of the study. Not only are there more blood vessels forming, FESEM results (Figure 8) show increased occurrence of vascular branching morphogenesis at earlier time points in samples containing $\mathrm{SiO}_{2}$ and $\mathrm{ZnO}$. Recently, silicon has been shown to play an important role in angiogenesis. One study demonstrated that silicate containing bioceramics can induce expression of kinase insert domain receptor (a receptor for vascular endothelial growth factor (VEGF)), basic fibroblast growth factor receptor 1 (a receptor for fibroblast growth factor (FGF)) and activin A receptor type II-like 1 (a receptor for transforming growth factor $\beta(T G F \beta)$ ) in human aortic endothelial cells [54]. VEGF, FGF and TGF $\beta$ are thought to be the main cytokines involved in angiogenesis. In the same study, a rabbit model was used to evaluate the angiogenic effects of the bioceramics where results demonstrated more and larger vessel formation as well as more branching. Zinc, has also demonstrated the ability to induce vascularization. In a study performed by Hanai et al. [55] zinc upregulated VEGF release from osteoblasts via increased FGF production.

The other half of the bone remodeling process is performed by osteoclasts. Osteoclasts produce TRAP, which creates an acidic microenvironment that favors the dissolution of calcium phosphate resulting in bone breakdown. In a situation where the expedited repair of a defect site is essential, it is beneficial to mitigate the activity of osteoclasts. Results from TRAP staining (Figure 9) indicate equal TRAP production over the course of 16 weeks were relatively similar in both the pure and doped samples. While counter intuitive, other studies have shown zinc supplementation in vivo and in vitro actually causes TRAP activity to increase while still decreasing the amount of osteoclastic bone resorption $[41,56]$. It was found that zinc facilitates an inverse relationship between TRAP and carbonic anhydrase II (CAII) production in osteoclast cells. CAII is also a vital enzyme in the osteoclastic resorption of the extracellular matrix (ECM). It acts by catalyzing hydrolysis of $\mathrm{CO}_{2}$ to generate $\mathrm{HCO}^{-}{ }_{3}$ and $\mathrm{H}^{+}$for solubilizing the ECM inorganic phase of bone ECM. So, as $\mathrm{Zn}^{2+}$ osteoclast cells produce more TRAP, they are producing less CAII. Another study, however, showed that silica based nanoparticles were able to significantly reduce TRAP activity in osteoclasts [57], so it is possible that there is a dual effect taking place. On the $\mathrm{Zn}^{2+}$ side, TRAP activity is increasing while decreasing the CAII activity, and on the other side, $\mathrm{Si}^{4+}$ is decreasing the TRAP activity back to normal levels resulting in an overall net osteoclastic resorption decrease. Although this phenomenon can be explained in terms of past research, it would be difficult to definitively define the relationship between $\mathrm{SiO}_{2}$ and $\mathrm{ZnO}$ on osteoclastic bone resorption from this study alone. More research is needed to better understand these relationships.

In addition to understanding the biological effects of the scaffolds in vivo, it is also important to understand the degradation kinetics of the material. AAS was performed (Figure 10) on urine collected from the control group during the course of the 16 week study to determine if either scaffold had degradation rates that were too high. It was found that urine levels of $\mathrm{Ca}^{2+}, \mathrm{Zn}^{2+}$ and $\mathrm{Si}^{4+}$ were nearly identical, indicating that if there were an excess of ions due to scaffold degradation, they were not excreted renally. These results would indicate that degradation of the scaffolds did not reach a level that the body could not effectively utilize. 


\subsection{Conclusion}

The goal of this research was to demonstrate that the addition of bone-important trace elements, such as zinc and silicon, to calcium phosphate scaffolds could add the supplementary benefit of osteoinduction and angiogenesis. In this study, 3D printing was used to fabricate pure and $\mathrm{SiO}_{2} / \mathrm{ZnO}$ doped tricalcium phosphate scaffolds with a designed porosity. Scaffolds were then implanted into a bicortical femur defect in a murine model. After 4 weeks, mechanical interlocking between the implant and the host tissue was stronger than the force it took to fracture the bone for both pure and doped samples. Goldner's trichrome histology, collagen I and osteocalcin immunostaining all indicated increased bone formation and maturation in doped samples over the course of 16 weeks. vonWillebrand Factor staining and FESEM micrographs also indicated an affinity for increased neovascularization in doped samples when compared to their pure counterparts. The addition of dopants did not affect the dissolution properties in vivo of the scaffold. Overall results, then, confirm that the addition of $\mathrm{SiO}_{2}$ and $\mathrm{ZnO}$ may be able to provide robust osteoinductive capabilities to $\mathrm{CaP}$ bone replacement materials without the need for biologics or pharmacologics.

\section{Acknowledgments}

Authors would like to acknowledge financial support from the National Institutes of Health, NIBIB (Grant \# NIHR01-EB-007351). The authors wish to also acknowledge the the School of Mechanical and Materials Engineering as well as the Franceschi Microscopy \& Imaging Center. The authors would like to thank Neal Davies (College of Pharmacy, WSU) for the lab space used for all animal surgeries.

\subsection{References}

1. Brown, PW. Encyclopedia of Materials: Science and Technology. Second Edition. Elsevier; Oxford: 2001. Calcium Phosphates in Biomedical Engineering; p. 893-7.

2. Bose S, Tarafder S. Calcium Phosphate Ceramic Systems in Growth Factor and Drug Delivery for Bone Tissue Engineering-A Review. Acta Biomaterialia. 2012; 8:1401-21. [PubMed: 22127225]

3. Yamada M, Shiota M, Yamashita Y, et al. Histological and histomorphometrical comparative study of the degradation and osteoconductive characteristics of $\alpha$ - and $\beta$ - tricalcium phosphate in block grafts. Journal of Biomedical Materials Research Part B: Applied Biomaterials. 2007; 82B:139-48.

4. Davies JE. Mechanisms of endosseous integration. Int J Prosthodont. 1998; 11:391-401. [PubMed: 9922731]

5. Hollister SJ. Porous scaffold design for tissue engineering. Nature Materials. 2005; 4:518-24.

6. Franco J, Launey AP, Tomsia ES. Direct write assembly of calcium phosphate scaffolds using a water-based hydrogel. Acta. 2010; 6:218-28.

7. Vorndran E, Klarner M, Klammert U, et al. 3D Powder Printing of $\beta$-Tricalcium Phosphate Ceramics Using Different Strategies. Advanced Engineering Materials. 2008; 10:B67-B71.

8. Khalyfa A, Vogt S, Weisser J, et al. Development of a new calcium phosphate powder-binder system for the 3D printing of patient specific implants. Journal of Materials Science: Materials in Medicine. 2007; 18:909-16. [PubMed: 17216579]

9. Fielding GA, Bandyopadhyay A, Bose $\mathrm{S}$. Effects of $\mathrm{SiO} 2$ and $\mathrm{ZnO}$ doping on mechanical and biological properties of 3D printed TCP scaffolds. Dental Materials. 2012; 28:113-22. [PubMed: 22047943]

10. Albrektsson T, Johansson C. Osteoinduction, osteoconduction and osseointegration. Eur Spine J. 2001; 10(Suppl 2):S96-101. [PubMed: 11716023]

11. Habibovic P, Yuan H, van der Valk CM, et al. 3D microenvironment as essential element for osteoinduction by biomaterials. Biomaterials. 2005; 26:3565-75. [PubMed: 15621247]

12. Chan O, Coathup MJ, Nesbitt A, et al. The effects of microporosity on osteoinduction of calcium phosphate bone graft substitute biomaterials. Acta Biomaterialia. 2012; 8:2788-94. [PubMed: 22475784] 
13. Otsuki B, Takemoto M, Fujibayashi S, et al. Pore throat size and connectivity determine bone and tissue ingrowth into porous implants: Three-dimensional micro-CT based structural analyses of porous bioactive titanium implants. Biomaterials. 2006; 27:5892-900. [PubMed: 16945409]

14. Li B, Liao X, Zheng L, et al. Effect of nanostructure on osteoinduction of porous biphasic calcium phosphate ceramics. Acta Biomaterialia. 2012; 8:3794-804. [PubMed: 22729020]

15. Matsumoto T, Okazaki M, Nakahira A, et al. Modification of apatite materials for bone tissue engineering and drug delivery carriers. Current medicinal chemistry. 2007; 14:2726-33. [PubMed: 17979722]

16. Yoshinari M, Oda Y, Inoue T, et al. Bone response to calcium phosphate-coated and bisphosphonate-immobilized titanium implants. Biomaterials. 2002; 23:2879-85. [PubMed: 12069328]

17. Martin I, Muraglia A, Campanile G, et al. Fibroblast Growth Factor-2 Supports ex Vivo Expansion and Maintenance of Osteogenic Precursors from Human Bone Marrow. Endocrinology. 1997; 138:4456-4462. [PubMed: 9322963]

18. Lan Levengood SK, Polak SJ, Poellmann MJ, et al. The effect of BMP-2 on micro- and macroscale osteointegration of biphasic calcium phosphate scaffolds with multiscale porosity. Acta Biomaterialia. 2010; 6:3283-91. [PubMed: 20176148]

19. Bohner M. Silicon-substituted calcium phosphates-a critical view. Biomaterials. 2009; 30:6403-6. [PubMed: 19695699]

20. Ito A, Kawamura H, Miyakawa S, et al. Resorbability and solubility of zinc-containing tricalcium phosphate. Journal of Biomedical Materials Research. 2002; 60:224-31. [PubMed: 11857428]

21. Bandyopadhyay A, Bernard S, Xue W, et al. Calcium Phosphate-Based Resorbable Ceramics: Influence of $\mathrm{MgO}, \mathrm{ZnO}$, and $\mathrm{SiO} 2$ Dopants. Journal of the American Ceramic Society. 2006; 89:2675-88.

22. Bose $\mathrm{S}$, Tarafder $\mathrm{S}$, Banerjee SS, et al. Understanding in vivo response and mechanical property variation in $\mathrm{MgO}, \mathrm{SrO}$ and $\mathrm{SiO} 2$ doped $\beta$-TCP. Bone. 2011; 48:1282-90. [PubMed: 21419884]

23. Banerjee SS, Tarafder S, Davies NM, et al. Understanding the influence of $\mathrm{MgO}$ and $\mathrm{SrO}$ binary doping on the mechanical and biological properties of [beta]-TCP ceramics. Acta Biomaterialia. 2010; 6:4167-74. [PubMed: 20493283]

24. Obata A, Tokuda S, Kasuga T. Enhanced in vitro cell activity on silicon-doped vaterite/poly (lactic acid) composites. Acta Biomaterialia. 2009; 5:57-62. [PubMed: 18786869]

25. Mastrogiacomo M, Papadimitropoulos A, Cedola A, et al. Engineering of bone using bone marrow stromal cells and a silicon-stabilized tricalcium phosphate bioceramic: evidence for a coupling between bone formation and scaffold resorption. Biomaterials. 2007; 28:1376-84. [PubMed: 17134749]

26. Pietak AM, Reid JW, Stott MJ, et al. Silicon substitution in the calcium phosphate bioceramics. Biomaterials. 2007; 28:4023-32. [PubMed: 17544500]

27. Eisinger JCD. Effects of silicon, fluoride, etidronate and magnesium on bone mineral density: a retrospective study. Magnes Res. 1993; 6:247-9. [PubMed: 8292498]

28. Botelho CM, Brooks RA, Best SM, et al. Human osteoblast response to silicon-substituted hydroxyapatite. J Biomed Mater Res A. 2006; 79:723-30. [PubMed: 16871624]

29. Yamaguchi M. Role of nutritional zinc in the prevention of osteoporosis. Molecular and Cellular Biochemistry. 2009; 338:241-54. [PubMed: 20035439]

30. Yamaguchi M, Weitzmann MN. Zinc stimulates osteoblastogenesis and suppresses osteoclastogenesis by antagonizing NF-кB activation. Mol Cell Biochem. 2011; 355:179-86. [PubMed: 21533765]

31. Kwun IS, Cho YE, Lomeda RAR, et al. Zinc deficiency suppresses matrix mineralization and retards osteogenesis transiently with catch-up possibly through Runx 2 modulation. Bone. 2010; 46:732-41. [PubMed: 19913120]

32. Yamaguchi M, Goto M, Uchiyama S, et al. Effect of zinc on gene expression in osteoblastic MC3T3-E1 cells: enhancement of Runx2, OPG, and regucalcin mRNA expressions. Molecular and Cellular Biochemistry. 2008; 312:157-66. [PubMed: 18327666] 
33. Reginster J, Saltman O, Franchimont P. Trace elements and postmenopausal osteoporosis: a preliminary study of decreased serum manganese. Journal of Nutrition. 1981; 111:1641-7. [PubMed: 7277041]

34. Xue W, Dahlquist K, Banerjee A, et al. Synthesis and characterization of tricalcium phosphate with $\mathrm{Zn}$ and Mg based dopants. Journal of Materials Science: Materials in Medicine. 2008; 19:2669-77. [PubMed: 18270806]

35. Egan KP, Brennan TA, Pignolo RJ. Bone histomorphometry using free and commonly available software. Histopathology. 2012; 61:1168-73. [PubMed: 22882309]

36. Wang Q, Chen Q, Zhu J, et al. Effects of pore shape and porosity on the properties of porous LNKN ceramics as bone substitute. Materials Chemistry and Physics. 2008; 109:488-91.

37. Van Bael S, Chai YC, Truscello S, et al. The effect of pore geometry on the in vitro biological behavior of human periosteum-derived cells seeded on selective laser-melted Ti6A14V bone scaffolds. Acta Biomaterialia. 2012; 8:2824-34. [PubMed: 22487930]

38. Bohner M, van Lenthe GH, Grünenfelder S, et al. Synthesis and characterization of porous betatricalcium phosphate blocks. Biomaterials. 2005; 26:6099-105. [PubMed: 15885772]

39. Tarafder S, Balla VK, Davies NM, et al. Microwave-sintered 3D printed tricalcium phosphate scaffolds for bone tissue engineering. Journal of Tissue Engineering and Regenerative Medicine. 2012 doi:10.1002/term.555.

40. Polak SJ, Levengood SKL, Wheeler MB, et al. Analysis of the roles of microporosity and BMP-2 on multiple measures of bone regeneration and healing in calcium phosphate scaffolds. Acta Biomaterialia. 2011; 7:1760-71. [PubMed: 21199692]

41. Hadley KB, Newman SM, Hunt JR. Dietary zinc reduces osteoclast resorption activities and increases markers of osteoblast differentiation, matrix maturation, and mineralization in the long bones of growing rats. The Journal of nutritional biochemistry. 2010; 21:297-303. [PubMed: 19369052]

42. Hie M, Iitsuka N, Otsuka T, et al. Zinc deficiency decreases osteoblasts and osteoclasts associated with the reduced expression of Runx2 and RANK. Bone. 2011; 49:1152-9. [PubMed: 21893222]

43. Matsko NB, Žnidaršič N, Letofsky-Papst I, et al. Silicon: The key element in early stages of biocalcification. Journal of Structural Biology. 2011; 174:180-6. [PubMed: 20932909]

44. Hing KA, Revell PA, Smith N, et al. Effect of silicon level on rate, quality and progression of bone healing within silicate-substituted porous hydroxyapatite scaffolds. Biomaterials. 2006; 27:501426. [PubMed: 16790272]

45. Bjerre L, Bünger CE, Kassem M, et al. Flow perfusion culture of human mesenchymal stem cells on silicate-substituted tricalcium phosphate scaffolds. Biomaterials. 2008; 29:2616-27. [PubMed: 18374976]

46. Gupta G, Kirakodu S, El-Ghannam A. Effects of exogenous phosphorus and silicon on osteoblast differentiation at the interface with bioactive ceramics. Journal of Biomedical Materials Research Part A. 2010; 95A:882-90. [PubMed: 20845489]

47. Wiesmann HP, Meyer U, Plate U, et al. Aspects of Collagen Mineralization in Hard Tissue Formation. In: International Review of Cytology. Elsevier. 2004:121-56.

48. Hie M, Tsukamoto I. Administration of zinc inhibits osteoclastogenesis through the suppression of RANK expression in bone. European Journal of Pharmacology. 2011; 668:140-6. [PubMed: 21806983]

49. Seaborn CD, Nielsen FH. Silicon deprivation decreases collagen formation in wounds and bone, and ornithine transaminase enzyme activity in liver. Biol Trace Elem Res. 2002; 89:251-61. [PubMed: 12462748]

50. Sommer B, Bickel M, Hofstetter W, et al. Expression of matrix proteins during the development of mineralized tissues. Bone. 1996; 19:371-80. [PubMed: 8894143]

51. Yamaguchi M, Hashizume M. Effect of $\beta$-alanyl-L-histidinato zinc on protein components in osteoblastic MC3T3-El cells: Increase in osteocalcin, insulin-like growth factor-I and transforming growth factor- $\beta$. Molecular and Cellular Biochemistry. 1994; 136:163-9. [PubMed: 7845370]

52. Varanasi V, Saiz E, Loomer P, et al. Enhanced osteocalcin expression by osteoblast-like cells (MC3T3-E1) exposed to bioactive coating glass $\left(\mathrm{SiO}_{2}-\mathrm{CaO}-\mathrm{P}_{2} \mathrm{O}_{5}-\mathrm{MgO}-\mathrm{K}_{2} \mathrm{O}-\mathrm{Na}_{2} \mathrm{O}\right.$ system) ions. Acta Biomaterialia. 2009; 5:3536-47. [PubMed: 19497391] 
53. Barradas AMC, Fernandes HAM, Groen N, et al. A calcium-induced signaling cascade leading to osteogenic differentiation of human bone marrow-derived mesenchymal stromal cells. Biomaterials. 2012; 33:3205-15. [PubMed: 22285104]

54. Zhai W, Lu H, Chen L, et al. Silicate bioceramics induce angiogenesis during bone regeneration. Acta Biomater. 2012; 8:341-9. [PubMed: 21964215]

55. Hanai Y, Tokuda H, Yasuda E, et al. Up-regulation by zinc of FGF-2-induced VEGF release throughenhancing p44/p42 MAP kinase activation in osteoblasts. Life sciences. 2006; 80:230-4. [PubMed: 17020773]

56. Holloway WR, Collier FM, Herbst RE, et al. Osteoblast-mediated effects of zinc on isolated rat osteoclasts: Inhibition of bone resorption and enhancement of osteoclast number. Bone. 1996; 19:137-42. [PubMed: 8853857]

57. Beck GR, Ha S-W, Camalier CE, et al. Bioactive silica-based nanoparticles stimulate boneforming osteoblasts, suppress bone-resorbing osteoclasts, and enhance bone mineral density in vivo. Nanomedicine: Nanotechnology, Biology and Medicine. 2012; 8:793-803. 


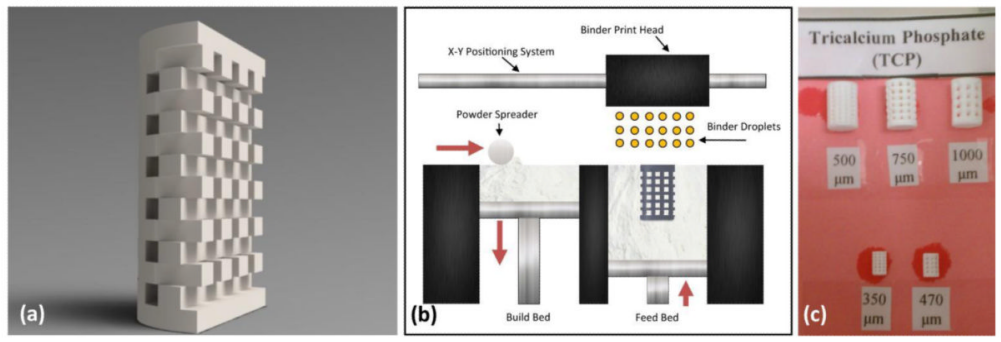

Figure 1.

(a) Rendered cross section of typical CAD file used for the 3D printing process. (b) Schematic diagram demonstrating 3D printing process. Red arrows indicate moving direction. (c) Actual scaffolds created by the Ex One printer. Numbers below the scaffold represent designed pore size. 

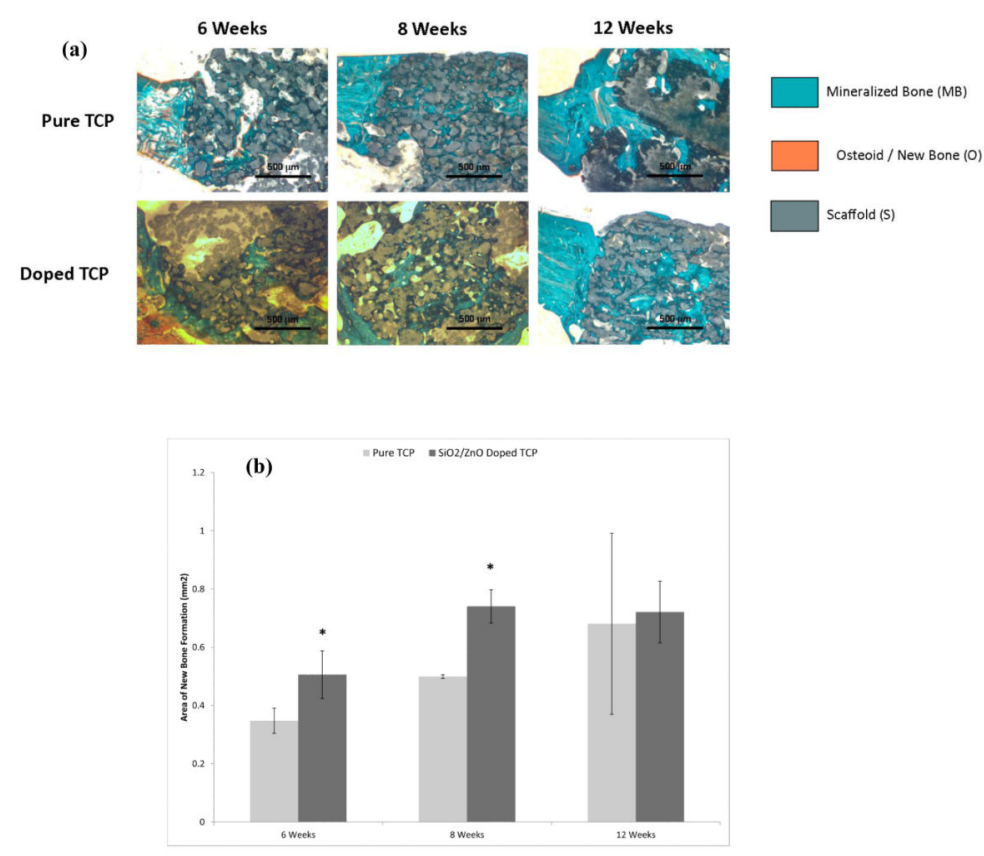

Figure 2.

(a) Confocal micrographs showing collagen I formation in sectioned implants over the course of 16 weeks. Green indicates collgen I, while blue indicates counterstain for cell nuclei. (b) Histomorphometry for collagen I labeled sections. ( $* P<0.1$, where $n=3$ ) 

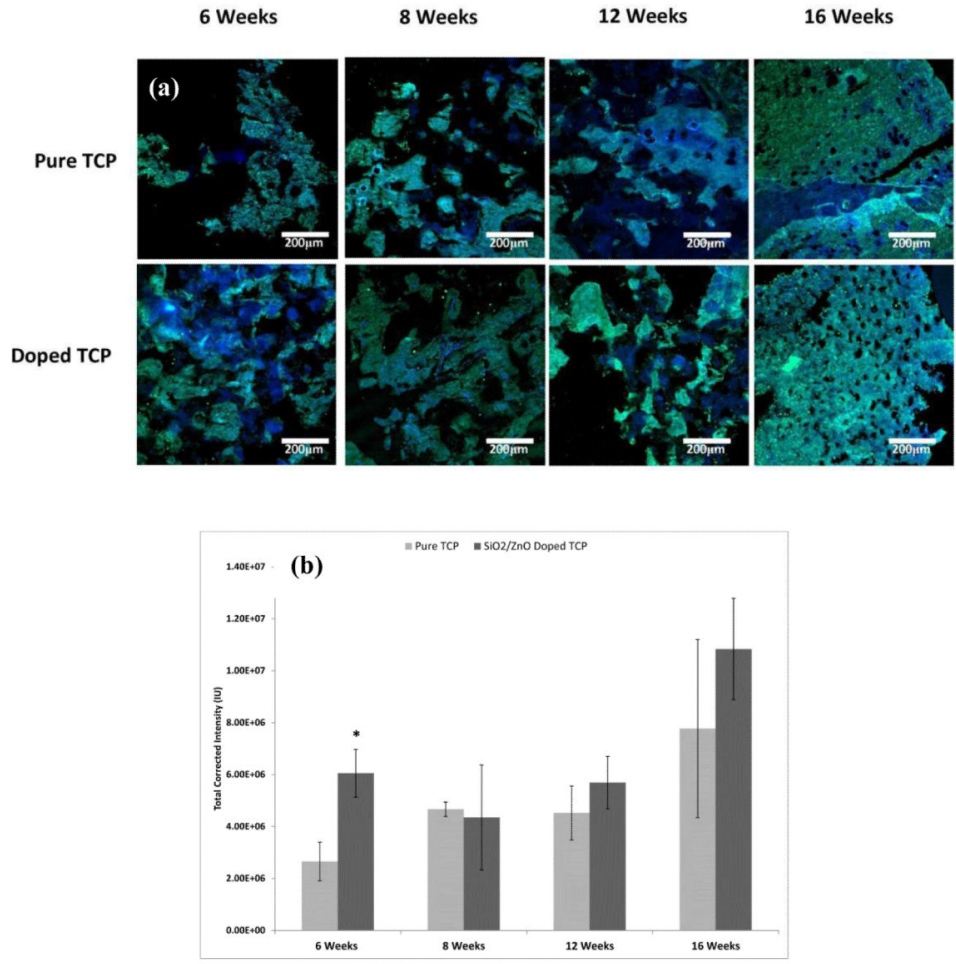

Figure 3.

(a) Confocal micrographs showing collagen I formation in sectioned implants over the course of 16 weeks. Green indicates collgen I, while blue indicates counterstain for cell nuclei. (b) Histomorphometry for collagen I labeled sections. ( ${ }^{\mathrm{P}}<0.1$, where $\mathrm{n}=3$ ) 

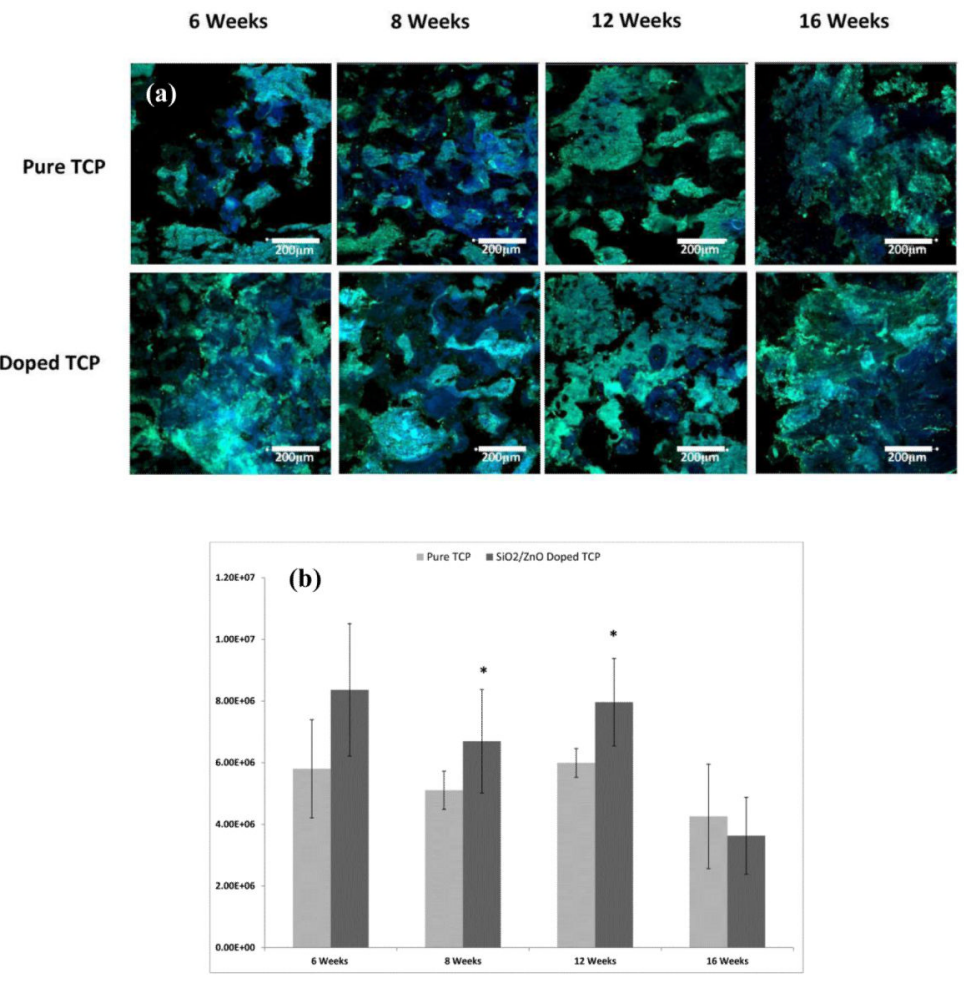

Figure 4.

(a) Confocal micrographs showing osteocalcin formation in sectioned implants over the course of 16 weeks. Green indicates osteocalcin, while blue indicates counterstain for cell nuclei. (b) Histomorphometry for osteocalcin labeled sections. ( $* \mathrm{P}<0.1$, where $\mathrm{n}=3$ ) 


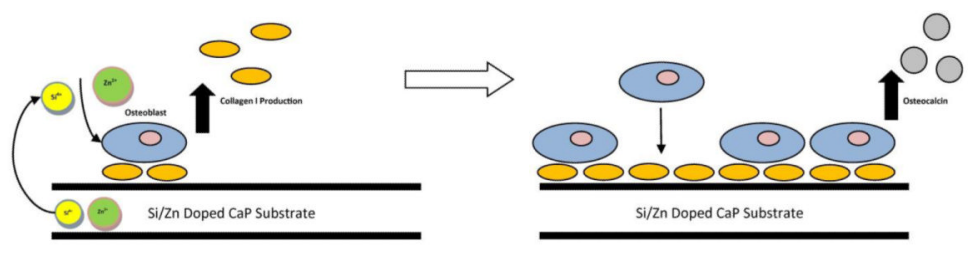

Figure 5.

Cartoon depicting effect of dopants on collagens and osteocalcin production. $\mathrm{Zn}^{2+}$ and $\mathrm{Si}^{4+}$ ions increase osteoblastic collagen matrix production. Increased collagen matrix leads to increased binding of osteoblasts to matrix integrins which activates the MAPK pathway. The MAPK pathways can eventually lead to the upregulation of osteocalcin expression. 

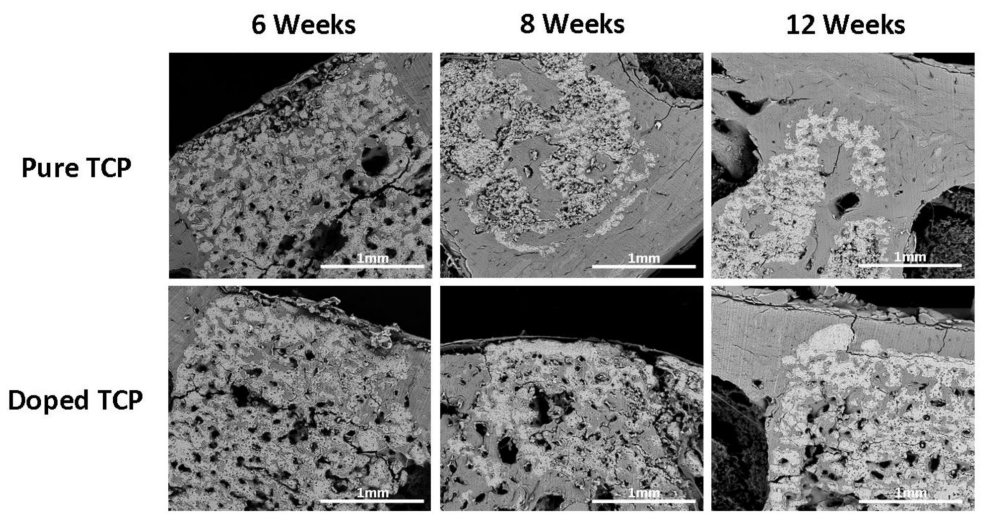

Figure 6.

BSE imaging of longitudinal cross sections of samples after 6, 8 and 12 weeks implantation. The lighter color indicates the calcium phosphate scaffold, while the darker areas indicate mineralized tissue. 


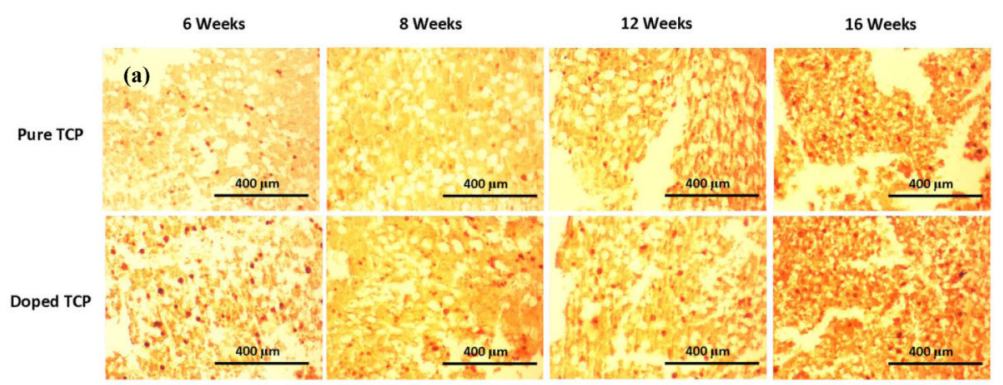

(b)

m Pure TCP $=$ SO2i/2nO Doped TCP

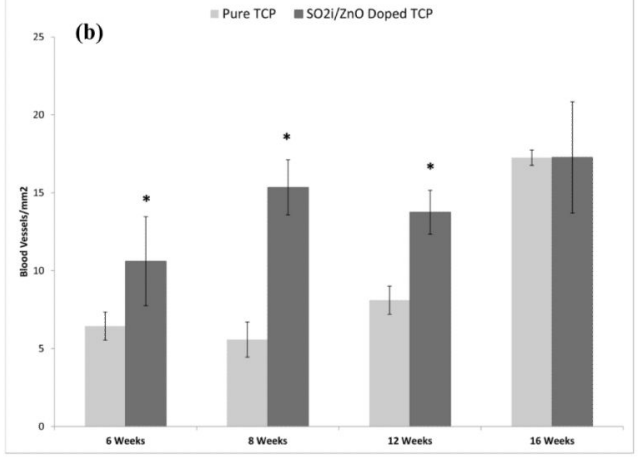

Figure 7.

(a) Light micrographs depicting vWF staining. The dark red spots within the sections are blood vessel. (b) Graph showing blood vessel density for each group over the course of 16 weeks. ( $* \mathrm{P}<0.1$, where $\mathrm{n}=3$ ) 


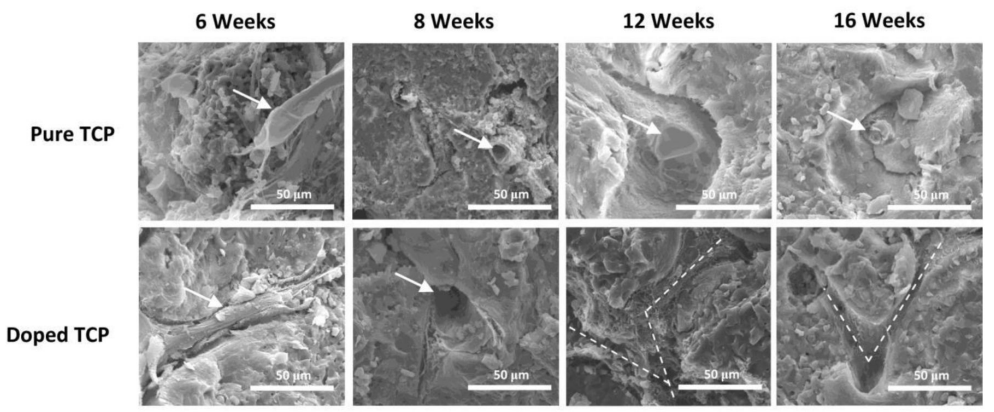

Figure 8.

FESEM micrographs showing blood vessel formation. Arrows indicate blood vessels. Dotted lines show vascular branching pathways formed in the samples. 


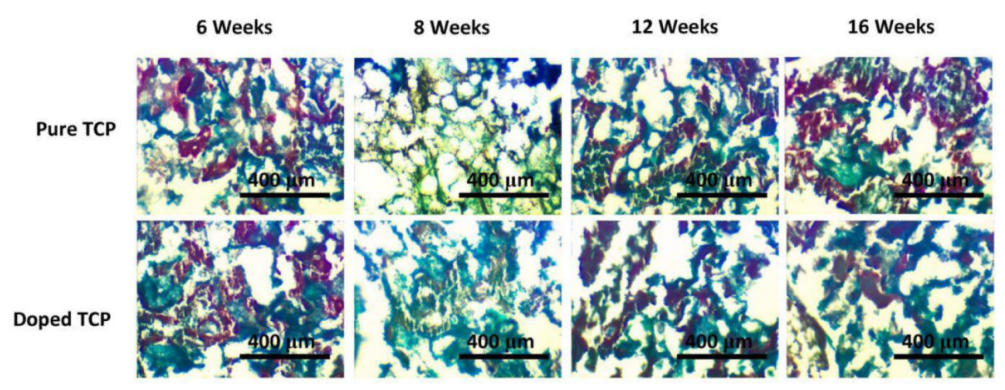

Figure 9.

TRAP staining in sectioned samples over the course of 16 weeks. The red color is indicative of TRAP activity and the green counterstain shows the tissue/scaffold. 


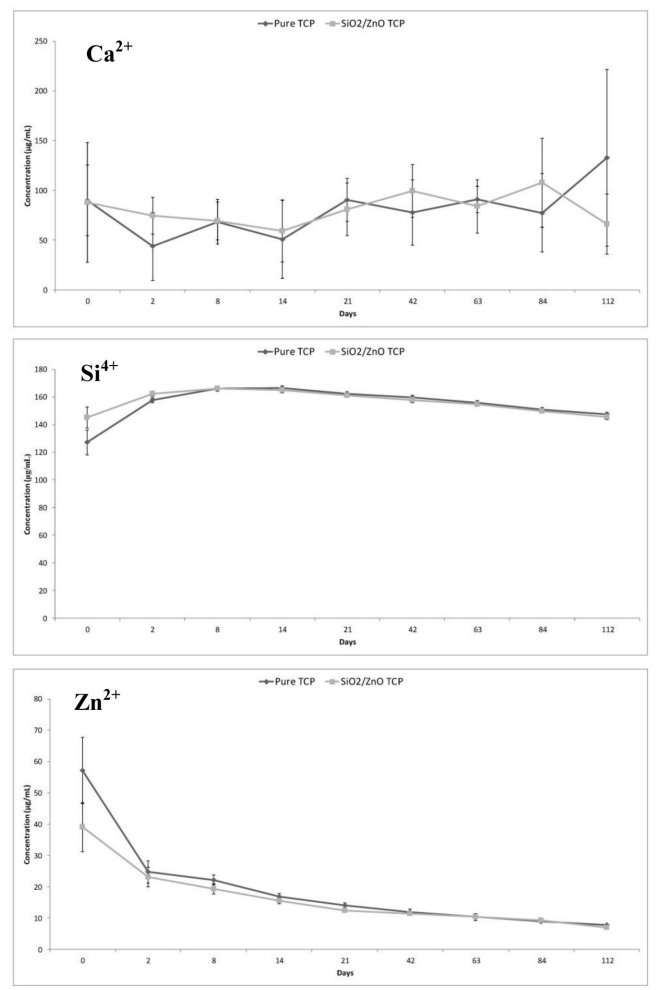

Figure 10.

AAS results for ion concentration in urine collected over 16 weeks. 